\title{
STABILIZATION OF NONLINEAR DISCRETE-TIME SYSTEMS VIA A DIGITAL COMMUNICATION CHANNEL
}

\author{
V. N. PHAT AND J. JIANG
}

Received 22 March 2004

We deal with the stabilization problem for a class of nonlinear discrete-time systems via a digital communication channel. We consider the case when the control input is to be transmitted via communication channels with a bit-rate constraint. Under an appropriate growth condition on the nonlinear perturbation, we establish sufficient conditions for the global and local stabilizability of semilinear and nonlinear discrete-time systems, respectively. A constructive method to design a feedback stabilizing controller is proposed.

\section{Introduction}

In recent years there has been a significant interest in the stabilization problem of dynamical systems (see, e.g., $[1,3,4,6,11,12,14,15,16]$ and the references therein). In the classical stabilization theory of dynamical systems, the standard assumption is that all data transmission required by the algorithm can be performed with infinite precision. However, in some new models, it is common to encounter situations where observation and control signals are sent via communication channel with a limited capacity. This problem may arise when large number of mobile units need to be controlled remotely by a single decision maker. Since the radio spectrum is limited, communication constraints are a real concern. All these new engineering applications motivated the development of a new chapter of control theory in which control and communication issues are combined together, and all the limitations of the communication channels are taken into account. Communication requirements, especially regarding bandwidth limits, are often challenging obstacles to control systems design (see, e.g., $[8,17,18]$ ). Furthermore, the focus has been on memoryless coding, in which the pant output is quantized without reference to its past. The paper [2] deals with a stabilization problem of a linear system with quantized state feedback and shows that if time-invariant system is passed through a fixed, memoryless quantizer, then controllability property of the system is impossible. In [7], the asymptotic stabilizability problem is investigated for a linear discrete-time system with a real-valued output when the controller, which may be nonlinear, receives observation data at a fixed known data rate. Petersen and Savkin $[9,10]$ present a feedback scheme for 
linear stabilization over a digital communication channel (DCC) using a nonlinear dynamic state feedback controller which can be applied to an arbitrary linear discrete-time system subject to standard assumptions of controllability and observability. However, the stabilization method used in these papers requires a significant amount of on-line computations and is hardly implementable in real time, especially in many control models described by a system of nonlinear equations.

In this paper, we investigate the stabilizability problem for a special class of nonlinear discrete-time control systems via DCC. The system considered in the paper consists of a linear discrete-time system and a linearly bounded nonlinear perturbation. Based on the state space quantization method used in $[2,7,8,9]$, we establish sufficient conditions for the global stabilizability of semilinear discrete-time systems under an appropriate growth condition on the nonlinear perturbation. The feedback stabilizing coder-controller is designed based on the measure of controllability matrix of the system. The approach enables us to derive a sufficient condition for the local stabilizability of a more general class of nonlinear discrete-time systems, where the right-hand side function is assumed to be smooth.

\section{Problem statement}

Consider a nonlinear control discrete-time system of the form

$$
x(k+1)=F(x(k), u(k)), \quad k \in \mathbb{Z}^{+}, \quad x(0)=x_{0},
$$

where $x(k) \in \mathbb{R}^{n}$ is the state; $u(k) \in \mathbb{R}^{m}$ is the control; $n \geq m, F(x, u): \mathbb{R}^{n} \times \mathbb{R}^{m} \rightarrow \mathbb{R}^{n}$ is a given nonlinear function; $\mathbb{Z}^{+}$is the set of all nonnegative integers; $\mathbb{R}^{n}$ is the $n$-dimensional Euclidean space.

Throughout the paper, $\mathbb{R}$ denotes the set of all real numbers; $\mathbb{R}^{n \times m}$ denotes the space of all real $(n \times m)$-matrices; $\|x\|_{\infty}$ and $\|A\|_{\infty}$ denote the infinity norms of the vector $x \in \mathbb{R}^{n}$ and the matrix $A=\left[a_{i j}\right] \in \mathbb{R}^{n \times m}$, defined as

$$
\|x\|_{\infty}=\max _{i=1, \ldots, n}\left|x_{i}\right|, \quad\|A\|_{\infty}=\max _{i=1, \ldots, n} \sum_{j=1}^{m}\left|a_{i j}\right| ;
$$

$B(a)$ denotes the closed hypercube in $\mathbb{R}^{n}$ with radius $a>0$ defined as $B(a)=\left\{x \in \mathbb{R}^{n}\right.$ : $\left.\|x\|_{\infty} \leq a\right\}$.

In control system (2.1), the way of communicating information from the measured state $x(k)$ to the control input $u(k)$ is via DCC. The feedback stabilization procedure for system (2.1) will involve two components. The first component, called the coder, takes the measured state signal $x(k)$ and produces a corresponding codeword $h(k)$ which is transmitted on the channel. The second component, called the controller, takes the received codeword $h(k)$ and produces the control input $u(k)$. The codeword $h(k)$ is restricted to a finite number of admissible codewords. The number of admissible codewords is determined by the data rate of the channel (see Figure 2.1). We use a multirate coder-controller in which the control input $u(k)$ is applied at every time step but a codeword is transmitted on the channel only once every $p$ time steps, that is, we assume that the coder and controller are defined by the following equations. 


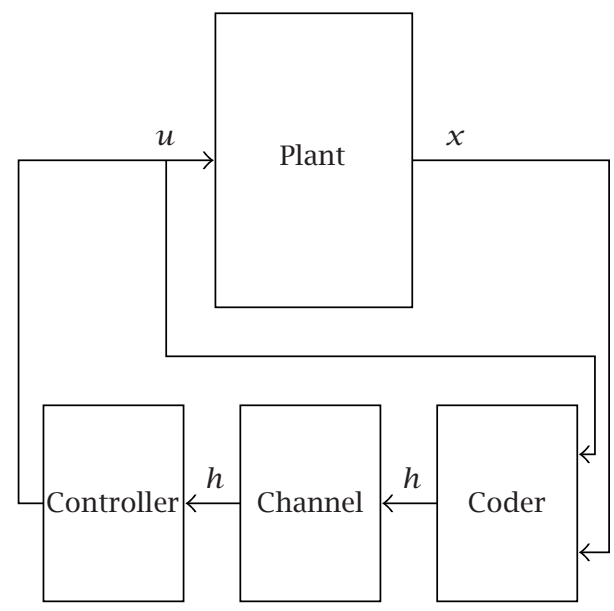

Figure 2.1. Coder-controller system.

(i) Coder

$$
a(p k+p)=g(a(p k), h(p k)), \quad a(0)=a_{0}, \quad h(p k)=H(a(p k), x(p k)) .
$$

(ii) Controller

$$
\begin{aligned}
a(p k+p)= & g(a(p k), h(p k)), \quad a(0)=a_{0}, \\
u(p k)= & g_{1}(a(p k), h(p k)), \\
u(p k+1)= & g_{2}(a(p k), h(p k)), \\
& \vdots \\
u(p k+p-1)= & g_{p}(a(p k), h(p k)),
\end{aligned}
$$

where $a(p k) \in \mathbb{R}$ is the quantization scaling which is updated every $p$ time steps. We assume that the coder and controller have available the initial value of the quantization scaling $a(0)=a_{0}$ and they haveaccess to the quantization scalings $a(p k)$ and $u(p k)$.

Definition 2.1. The system (2.1) is said to be globally stabilizable via a DCC, if there exists a coder-controller of the form (2.3)-(2.4) with a quantization scaling $a_{0}$, and for every initial condition $x(0)=x_{0}$, the corresponding solution $x(k)$ of the closed-loop system satisfies

$$
x(k) \longrightarrow 0 \text { as } k \longrightarrow \infty
$$

In this case it is also said that coder-controller (2.3)-(2.4) globally stabilizes system (2.1) via a DCC. If the above assertion holds for all $x_{0}$ belonging to some neighborhood of the origin in $\mathbb{R}^{n}$, it is said that coder-controller (2.3)-(2.4) locally stabilizes system (2.1) via DCC. 
The objective of this paper is to construct the coder-controller which globally (locally) stabilizes uncertain system (2.1) via DCC.

\section{Semilinear discrete-time systems and state quantization}

In this section, we start considering a semilinear discrete control system of the form

$$
x(k+1)=A x(k)+B u(k)+f(x(k), u(k)), \quad k=0,1,2, \ldots,
$$

where $A, B$ are constant matrices, $f(\cdot)$ is a nonlinear function. In order to describe and analyze our multirate control system, it is convenient to consider an equivalent singlerate system described as follows. For this, we use a multirate coder-controller in which the control input $u(k)$ is applied at every time step but a codeword is transmitted on the channel only once every $p$ time steps.

Let $p \geq 1$ be the smallest integer such that $\operatorname{rank}\left[B, A B, \ldots, A^{p-1} B\right]=n$. From the discretetime system (3.1), we have

$$
\begin{aligned}
& x(p k+1)= A x(p k)+B u(p k)+f(x(p k), u(p k)), \\
& x(p k+2)= A^{2} x(p k)+A B u(p k)+B u(p k+1) \\
&+A f(x(p k), u(p k))+f(x(p k+1), u(p k+1)), \\
& \vdots \\
& x(p k+i)= A^{i} x(p k)+\sum_{i=0}^{j-1} A^{j-i-1} B u(p k+i) \\
&+\sum_{i=0}^{j-1} A^{j-i-1} f(x(p k+i), u(p k+i)),
\end{aligned}
$$

or in the discrete $p$-delay time system

$$
x(p k+p)=\Phi x(p k)+G v(k)+\sum_{i=0}^{p-1} A^{p-i-1} f(x(p k+i), u(p k+i))
$$

where

$$
\begin{gathered}
\Phi=A^{p}, \quad G=\left[B, A B, \ldots, A^{p-1} B\right], \\
v(k)=[u(p k), u(p k+1), \ldots, u(p k+p-1)]^{T} .
\end{gathered}
$$

We denote $y(k)=x(p k)$. Then the system (3.3) is rewritten in the single-rate form

$$
y(k+1)=\Phi y(k)+G v(k)+W(y(k), v(k))
$$

where

$$
W(y(k), v(k)):=\sum_{i=0}^{p-1} A^{p-i-1} f(x(p k+i), u(p k+i))
$$


We quantize the state space of system (3.5) as follows. For consistency we denote $\bar{a}(k)=$ $a(p k), \bar{h}(k)=h(p k)$. Given sequence $\bar{a}(k)$, we quantize the state space of system (3.5) by dividing the set $B(\bar{a}(k))$ into $q^{n}$ hypercubes. For each $i \in\{1,2, \ldots, n\}$ we divide the corresponding state coordinate $y_{i} \in \mathbb{R}$ into $q$ intervals of the form

$$
\begin{gathered}
I_{1, i}(\bar{a}(k))=\left\{y_{i}:-\bar{a}(k) \leq y_{i}<-\bar{a}(k)+\frac{2 \bar{a}(k)}{q}\right\}, \\
\vdots \\
I_{q, i}(\bar{a}(k))=\left\{y_{i}: \bar{a}(k)-\frac{2 \bar{a}(k)}{q} \leq y_{i} \leq \bar{a}(k)\right\} .
\end{gathered}
$$

Thus, for any vector $y \in B(\bar{a}(k))$, there exist unique integers $i_{1}, i_{2}, \ldots, i_{n} \in\{1,2, \ldots, q\}$ such that $y \in I_{i, j}(\bar{a}(k))$. According to the integers $i_{1}, i_{2}, \ldots, i_{n}$, we define the vector

$$
\eta\left(i_{1}, i_{2}, \ldots, i_{n}\right):=\left(\begin{array}{c}
-\bar{a}(k)+\frac{\bar{a}(k)\left(2 i_{1}-1\right)}{q} \\
-\bar{a}(k)+\frac{\bar{a}(k)\left(2 i_{2}-1\right)}{q} \\
\vdots \\
-\bar{a}(k)+\frac{\bar{a}(k)\left(2 i_{n}-1\right)}{q}
\end{array}\right) .
$$

This vector is the center of the corresponding hypercube $I_{i, j}(\bar{a}(k))$ containing the original point $y$ and

$$
y \in \eta\left(i_{1}, i_{2}, \ldots, i_{n}\right)+B\left(\frac{\bar{a}(k)}{q}\right)
$$

Note that the region $\mathbb{R}^{n} \backslash B(\bar{a}(k))$ partition the state space into $q^{n}+1$ regions. Then, in our proposed coder-controller, for $y \in B(\bar{a}(k))$, the transmitted codeword will correspond to the integers $\left\{i_{1}, i_{2}, \ldots, i_{n}\right\}$. For $y \in \mathbb{R}^{n} \backslash B(\bar{a}(k))$ we assign the codeword $\{0\}$. Moreover, note that our method of quantization of the state space of system (3.5) depends on the scaling parameter $\bar{a}(k)$, which is available to both the controller and coder at any step time $k$, and the update law for $\bar{a}(k)$. Note that the regions $I_{i, j}$ together with the region $\mathbb{R}^{n} \backslash B(\bar{a}(k))$ partition the state space into $q^{n}+1$ regions (see Figure 3.1 ).

Remark 3.1. Note that the number $p>0$, as mentioned before, is defined as a finite time at which the linear discrete-time system $[A, B]$ is globally controllable. Following the wellknown controllability criterion of discrete-time system (see, e.g., $[5,13]$ ), this number is the smallest integer such that $\operatorname{rank}[A \mid B]=\left[A, B, \ldots, A^{p-1} B\right]=n$. Since the matrix $[A \mid B]$ has $(n \times n m)$-dimension, $n \geq m$, we have $p=1$, if matrix $B$ has full rank $m=n$. Otherwise, this number can be defined as the smallest number of the linearly independent columns of matrix $[A \mid B]$. 


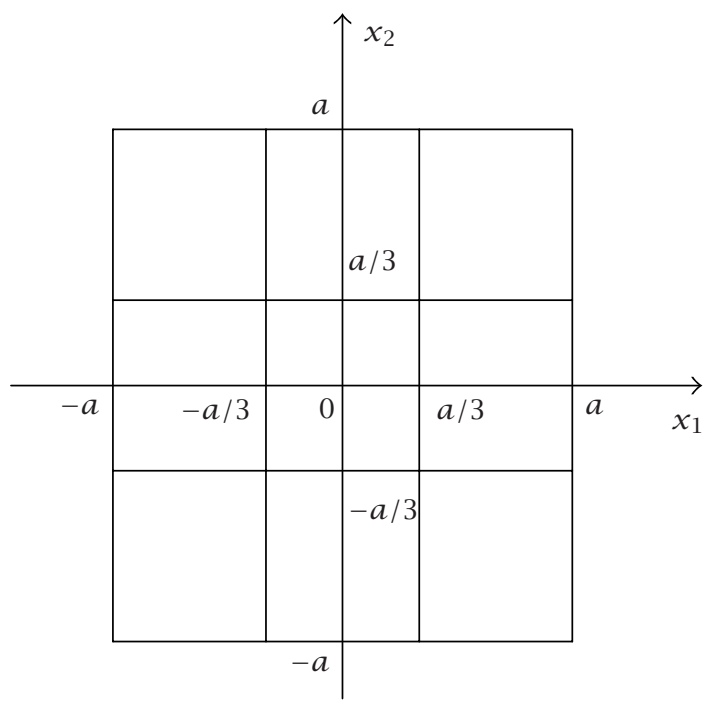

Figure 3.1. State quantization.

\section{Main results}

Consider the discrete-time system (3.1). Assume the following.

Assumption 4.1. The linear discrete-time system $[A, B]$ is globally controllable, that is, there is $p \geq 1$ such that $\operatorname{rank}\left[B, A B, \ldots, A^{p-1} B\right]=n$.

Assumption 4.2. The function $f(x, u)$ satisfies the following growth condition:

$$
\exists c, d>0:\|f(x, u)\| \leq c\|x\|+d\|u\|, \quad \forall(x, u) \in \mathbb{R}^{n} \times \mathbb{R}^{m} .
$$

Introduce the following notations:

$$
\begin{aligned}
& K:=G^{\prime}\left(G G^{\prime}\right)^{-1} \Phi, \quad \beta=\|B\|, \quad \alpha=\max \left\{\left\|A^{i}\right\|, i=0,1, \ldots, p-1\right\}, \\
& \gamma=\|K\|, \quad \eta=(\beta+d) \gamma \frac{q-1}{q}, \\
& \sigma= \begin{cases}c & \text { if } p=1, \\
c \alpha \sum_{i=1}^{p-1}\left[(\alpha+c)^{i}+\sum_{j=0}^{i}(\alpha+c)^{j} \eta\right] & \text { if } p>1,\end{cases} \\
& \xi=c \alpha q \sigma+\operatorname{d\alpha p} \gamma(q-1), \quad \lambda=\frac{\|\Phi\|+\xi}{q} \text {. }
\end{aligned}
$$

We first prove the stabilizability of single-rate system (3.5). For this, we construct the coder-controller of the form (2.3)-(2.4) for system (3.5) as follows. Consider the sequence 
$\bar{a}(k)$ satisfying

$$
\bar{a}(k+1)=\lambda a(k), \quad \bar{a}(0)=a_{0}, \quad \forall k \in \mathbb{Z}^{+},
$$

and for a given integer $q>1$, by the quantization method described in the previous section we quantize the state space of system (3.5) by diving the set $B(\bar{a}(k))$ into $q^{n}$ hypercubes of the form (3.7). Note that for any $k \in \mathbb{Z}^{+}$, if $y(k) \in B(\bar{a}(k))$, then there is a vector $\eta\left(i_{1}, i_{2}, \ldots, i_{n}\right) \in \mathbb{R}^{n}$ such that

$$
y(k) \in \eta\left(i_{1}, i_{2}, \ldots, i_{n}\right)+B\left(\frac{\bar{a}(k)}{q}\right) .
$$

For $y(k) \notin B(\bar{a}(k))$ we assign the codeword $\{0\}$.

(i) Coder

$$
\begin{aligned}
\bar{a}(k+1) & = \begin{cases}\lambda \bar{a}(k) & \text { if } y(k) \in B(\bar{a}(k)), \bar{a}(0)=a_{0}, \\
r \bar{a}(k) & \text { if } y(k) \notin B(\bar{a}(k)),\end{cases} \\
\bar{h}(k) & = \begin{cases}\left\{i_{1}, i_{2}, \ldots, i_{n}\right\} & \text { if } y(k) \in B(\bar{a}(k)), \\
\{0\} & \text { if } y(k) \notin B(\bar{a}(k)) .\end{cases}
\end{aligned}
$$

(ii) Controller

$$
\begin{aligned}
\bar{a}(k+1) & = \begin{cases}\lambda \bar{a}(k) & \text { if } \bar{h}(k) \neq\{0\}, \bar{a}(0)=a_{0}, \\
r \bar{a}(k) & \text { if } \bar{h}(k)=\{0\},\end{cases} \\
v(k) & = \begin{cases}-K \eta\left(i_{1}, i_{2}, \ldots, i_{n}\right) & \text { if } \bar{h}(k)=\left\{i_{1}, i_{2}, \ldots, i_{n}\right\}, \\
\{0\} & \text { if } \bar{h}(k)=\{0\},\end{cases}
\end{aligned}
$$

where the positive integer $r \in \mathbb{Z}^{+}$is chosen so that

$$
r>\|\Phi\|+c \alpha \sum_{i=0}^{p-1}(\alpha+c)^{i} .
$$

Theorem 4.3. Suppose that Assumptions 4.1 and 4.2 hold, and

$$
\lambda=\frac{\|\Phi\|+\xi}{q}<1
$$

Then the coder-controller (4.5)-(4.6) globally stabilizes single-rate system (3.5) via DCC.

Proof. The proof consists of two parts.

(A) We first prove that if there is an integer $k_{0} \in \mathbb{Z}^{+}$such that $y\left(k_{0}\right) \in B\left(\bar{a}\left(k_{0}\right)\right)$, then $y(k) \in B(\bar{a}(k))$, for all $k \geq k_{0}$. Indeed, if $y\left(k_{0}\right) \in B\left(\bar{a}\left(k_{0}\right)\right)$, then by the state space quantization of system (3.5), there is a vector $\eta\left(i_{1}, i_{2}, \ldots, i_{n}\right) \in \mathbb{R}^{n}$ such that

$$
y\left(k_{0}\right) \in \eta\left(i_{1}, i_{2}, \ldots, i_{n}\right)+B\left(\frac{\bar{a}\left(k_{0}\right)}{q}\right) .
$$


From (3.5) it follows that

$$
y\left(k_{0}+1\right) \in \Phi \eta\left(i_{1}, i_{2}, \ldots, i_{n}\right)+\Phi B\left(\frac{\bar{a}\left(k_{0}\right)}{q}\right)+G v\left(k_{0}\right)+W\left(y\left(k_{0}\right), v\left(k_{0}\right)\right) .
$$

Substituting the controller (4.6) in the last inclusion, we find

$$
\begin{aligned}
y\left(k_{0}+1\right) & \in \Phi \eta(\cdot)+\Phi B\left(\frac{\bar{a}\left(k_{0}\right)}{q}\right)-\Phi \eta(\cdot)+W\left(y\left(k_{0}\right), v\left(k_{0}\right)\right) \\
& =\Phi B\left(\frac{\bar{a}\left(k_{0}\right)}{q}\right)+W\left(y\left(k_{0}\right), v\left(k_{0}\right)\right)
\end{aligned}
$$

and hence

$$
\left\|y\left(k_{0}+1\right)\right\| \leq \frac{\|\Phi\|}{q} \bar{a}\left(k_{0}\right)+\left\|W\left(y\left(k_{0}\right), v\left(k_{0}\right)\right)\right\| .
$$

Using condition (4.1), we have

$$
\begin{aligned}
& \left\|W\left(y\left(k_{0}\right), v\left(k_{0}\right)\right)\right\| \\
& \quad \leq c \sum_{i=0}^{p-1}\left\|A^{p-i-1}\right\|\left\|x\left(p k_{0}+i\right)\right\|+d \sum_{i=0}^{p-1}\left\|A^{p-i-1}\right\|\left\|u\left(p k_{0}+i\right)\right\| .
\end{aligned}
$$

Since $\left\|u\left(p k_{0}+i\right)\right\| \leq\left\|v\left(k_{0}\right)\right\|$, and note that $\eta(\cdot)$ is the center of the hypercube $I_{i, j}(\cdot) \subset$ $B\left(\bar{a}\left(k_{0}\right)\right)$, we have

$$
\|\eta(\cdot)\| \leq \bar{a}\left(k_{0}\right)-\frac{\bar{a}\left(k_{0}\right)}{q}=\frac{q-1}{q} \bar{a}\left(k_{0}\right) .
$$

Moreover, from the system of discrete-time equations (3.3), we can verify by induction the following estimation:

$$
\left\|x\left(p k_{0}+i\right)\right\| \leq\left\{(\alpha+c)^{i}+\sum_{j=0}^{i-1}\left[(\alpha+c)^{j} \eta\right]\right\}\left\|x\left(p k_{0}\right)\right\|
$$

for all $i=1,2, \ldots, p-1, p>1$. Therefore,

$$
\left\|W\left(y\left(k_{0}\right), v\left(k_{0}\right)\right)\right\| \leq\left\{c \alpha \sigma+d \alpha \gamma \frac{q-1}{q}\right\}\left\|y\left(k_{0}\right)\right\| .
$$


Thus, we obtain that

$$
\begin{aligned}
\left\|y\left(k_{0}+1\right)\right\| & \leq \frac{\|\Phi\|}{q} \bar{a}\left(k_{0}\right)+\left[c \alpha \sigma+d \alpha \gamma \frac{q-1}{q}\right] a\left(k_{0}\right) \\
& =\frac{\|\Phi\|+\xi}{q} \bar{a}\left(k_{0}\right)=\lambda \bar{a}\left(k_{0}\right)=\bar{a}\left(k_{0}+1\right),
\end{aligned}
$$

which means that $y\left(k_{0}+1\right) \in B\left(\bar{a}\left(k_{0}+1\right)\right)$.

(B) We show that there is at least $k_{0} \in \mathbb{Z}^{+}$such that $y\left(k_{0}\right) \in B\left(\bar{a}\left(k_{0}\right)\right)$. Assume to the contrary that for all $k \in \mathbb{Z}^{+}, y(k) \notin B(\bar{a}(k))$. In this case, taking $v(k)=0$, from (3.5) it follows that

$$
\|y(k+1)\| \leq\|\Phi y(k)\|+\|W(y(k), 0)\|, \quad k=0,1,2, \ldots
$$

Hence

$$
\|W(y(k), 0)\| \leq c \sum_{i=0}^{p-1}\left\|A^{p-i-1}\right\|\|x(p k+i)\|, \quad k \in \mathbb{Z}^{+} .
$$

On the other hand, from the system (3.1) with $v(k)=0$, we can verify by induction that

$$
\|x(p k+i)\| \leq(\alpha+c)^{i}\|y(k)\|, \quad k \in \mathbb{Z}^{+},
$$

then

$$
\|y(k+1)\| \leq\left[\|\Phi\|+c \alpha \sum_{i=0}^{p-1}(c+\alpha)^{i}\right]\|y(k)\|, \quad k \in \mathbb{Z}^{+} .
$$

Therefore

$$
\|y(k)\| \leq\left[\|\Phi\|+c \alpha \sum_{i=0}^{p-1}(c+\alpha)^{i}\right]^{k}\left\|y_{0}\right\|, \quad k \in \mathbb{Z}^{+} .
$$

By the definition of the sequence $\bar{a}(k)$, for the case $y(k) \notin B(\bar{a}(k))$, taking any $\bar{a}(0)=a_{0}>$ 0 , we have $\bar{a}(k)=r^{k} a_{0}, k \in \mathbb{Z}^{+}$, and hence

$$
\frac{\|y(k)\|}{\bar{a}(k)} \leq\left[\frac{\|\Phi\|+c \alpha \sum_{i=0}^{p-1}(c+\alpha)^{i}}{r}\right]^{k} \frac{1}{a_{0}}, \quad k \in \mathbb{Z}^{+} .
$$

Using the condition (4.7) gives

$$
\frac{\|y(k)\|}{\bar{a}(k)} \longrightarrow 0 \quad \text { as } k \longrightarrow \infty .
$$

Thus, there is a number $k_{0}$ such that $y\left(k_{0}\right) \in B\left(\bar{a}\left(k_{0}\right)\right)$, which contradicts the contrary assumption.

Finally, combining (A) and (B) gives

$$
\exists k_{0} \in \mathbb{Z}^{+}: y(k) \in B(\bar{a}(k)), \quad \text { where } \bar{a}(k+1)=\lambda \bar{a}(k), \forall k \geq k_{0} .
$$




\section{Stabilization of discrete-time systems}

This means that

$$
\|y(k)\| \leq \bar{a}(k)=\lambda^{k} a_{0} \quad \forall k \geq k_{0}
$$

Taking condition (4.8) into account, we have $y(k) \rightarrow 0$ as $k \rightarrow \infty$, which completes the proof of the theorem.

Theorem 4.4. Assume that Assumptions 4.1, 4.2, and the condition (4.8) hold. Then the multirate state feedback coder-controller of the form (2.3)-(2.4) corresponding to the singlerate coder-controller (4.5)-(4.6) globally stabilizes the system (3.1) via DCC.

Proof. Let $x_{0} \in \mathbb{R}^{n}$ be an arbitrary initial state. Let $x(k)$ be the solution of system (2.1) with the multirate coder-controller corresponding to the coder-controller (4.5)-(4.6). By Theorem 4.3,

$$
\|x(p k)\| \leq \lambda^{p k}\left\|x_{0}\right\| \quad \forall k \in \mathbb{Z}^{+}
$$

and hence the solution $x(\cdot)$ at the time rate $p$ goes to 0 as $k$ goes to $\infty$. For any time instant $p k+j, j=1,2, \ldots, p-1$, the solution $x(p k+j)$, using the estimation (4.13), satisfies the following estimation:

$$
\|x(p k+j)\| \leq M\|x(p k)\|
$$

for some positive number $M>0$, and hence the solution also goes to 0 , because of $x(p k) \rightarrow$ 0 . The proof of the theorem is completed.

We now consider nonlinear discrete-time system (2.1), where the nonlinear function $F(x, u)$ is continuously differentiable in $(x, u)$ and $F(0,0)=0$. In this case, nonlinear function $F(x, u)$ can be linearized as

$$
F(x, u)=A x+B u+f(x, u)
$$

where

$$
A=\frac{\partial F(0,0)}{\partial x}, \quad B=\frac{\partial F(0,0)}{\partial u}
$$

and the nonlinear perturbation function $f(x, u)$ satisfies the following bounded growth condition:

$$
\forall \epsilon>0, \quad \exists \delta>0:\|f(x, u)\|<\epsilon(\|x\|+\|u\|),
$$

for all $\|x\|+\|u\|<\delta$. Using the proof of Theorem 4.3 letting $c=d=\epsilon$, if $\|\Phi\| / q<1$, we can choose a number $\epsilon>0$ such that

$$
\frac{\|\Phi\|}{q}+\epsilon\left[\alpha \sigma+\alpha p \gamma \frac{q-1}{q}\right]<1
$$


Therefore, as a consequence of Theorem 4.4, we obtain the following result, which gives a sufficient condition for the local stabilizability of nonlinear system (2.1).

Theorem 4.5. Assume that $F(x, u)$ is continuously differentiable in $(x, u), F(0,0)=0$, and the system $[A, B]$ is globally controllable. If

$$
\frac{\|\Phi\|}{q}<1
$$

then the multirate state feedback coder-controller of the form (2.3)-(2.4) corresponding to the single-rate coder-controller (4.5)-(4.6) locally stabilizes nonlinear discrete-time system (2.1) via DCC.

Illustrative example. Consider the following discrete-time system:

$$
x(k+1)=1.2 x(k)+u(k)+f(x(k), u(k)),
$$

where

$$
f(x(k), u(k))=0.2 x(k) \sin u(k)+0.5 u(k) \cos ^{2} x(k) .
$$

We have $c=0.2, d=0.5$. In this case $p=1, q=3$. With simple calculations, we find

$$
\begin{gathered}
K:=G^{\prime}\left(G G^{\prime}\right)^{-1} \Phi=1.2, \quad \beta=\|B\|=1, \quad \alpha=1.2, \\
\gamma=\|K\|=1.2, \quad \eta=(\beta+d) \gamma \frac{q-1}{q}=1.2, \\
\sigma=c=0.2, \quad \xi=c \alpha q \sigma+\operatorname{d\alpha p} \gamma(q-1)=1.5840, \\
\lambda=\frac{\|\Phi\|+\xi}{q}=0.928<1, \quad\|\Phi\|+c \alpha(\alpha+c)^{0}=1.44 .
\end{gathered}
$$

Taking $r=2$, the coder and controller of system (4.34) is constructed as follows.

(i) Coder

$$
\begin{aligned}
& a(k+1)= \begin{cases}0.928 a(k), & |x(k)| \leq a(k), \\
2 a(k), & |x(k)|>a(k),\end{cases} \\
& h(k)= \begin{cases}0, & |x(k)|>a(k), \\
1, & x(k) \in\left[-a(k),-\frac{a(k)}{3}\right], \\
2, & x(k) \in\left[-\frac{a(k)}{3}, \frac{a(k)}{3}\right], \\
3, & x(k) \in\left[\frac{a(k)}{3}, a(k)\right] .\end{cases}
\end{aligned}
$$



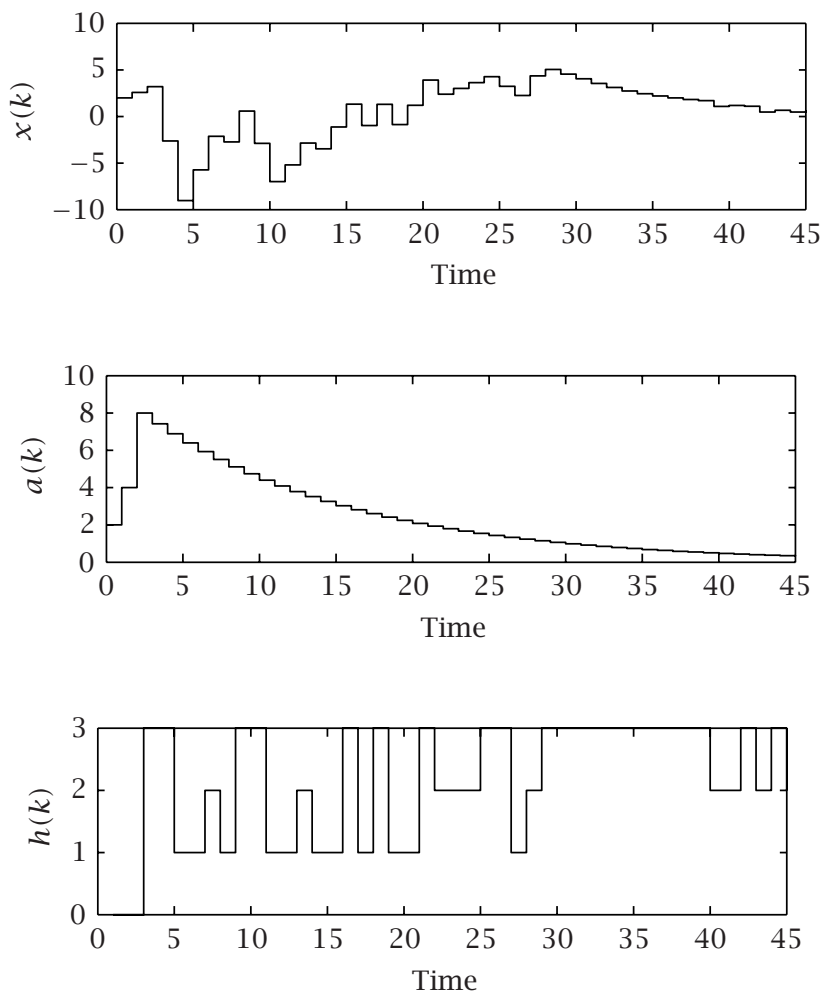

Figure 4.1. Simulation result.

(ii) Controller

$$
\begin{aligned}
a(k+1) & = \begin{cases}0.928 a(k), & h(k) \neq 0, \\
2 a(k), & h(k)=0,\end{cases} \\
u(k) & = \begin{cases}-1.2 \eta(k), & h(k)=\{1,2,3\}, \\
0, & h(k)=0,\end{cases} \\
\eta(k) & = \begin{cases}-\frac{2 a(k)}{3}, & h(k)=1, \\
0, & h(k)=2, \\
\frac{2 a(k)}{3}, & h(k)=3 .\end{cases}
\end{aligned}
$$

The simulation result of the above coder-controller applied to system (4.34) with $x(0)=2$ is shown in Figure 4.1. 


\section{Acknowledgments}

This work was done during the first author's stay at the Department of Electrical Engineering and Telecommunications, University of New South Wales, Sydney, Australia. The paper was supported by the Australian Research Council and in part by the National Basic Program in Natural Sciences, Vietnam. The authors would like to thank the anonymous referees for their valuable remarks and comments, which have improved our paper.

\section{References}

[1] R. W. Brockett and D. Liberzon, Quantized feedback stabilization of linear systems, IEEE Trans. Automat. Control 45 (2000), no. 7, 1279-1289.

[2] D. F. Delchamps, Stabilizing a linear system with quantized state feedback, IEEE Trans. Automat. Control 35 (1990), no. 8, 916-924.

[3] G. Escobar, R. Ortega, and H. Sira-Ramirez, Output-feedback global stabilization of a nonlinear benchmark system using a saturated passivity-based controller, IEEE Trans. Contr. Syst. Tech. 7 (1999), 289-293.

[4] G. Feng and J. Ma, Quadratic stabilization of uncertain discrete-time fuzzy dynamic system, IEEE Trans. Circuits Systems I Fund. Theory Appl. 48 (2001), 1337-1344.

[5] J. Klamka, Controllability of Dynamical Systems, Mathematics and Its Applications (East European Series), vol. 48, Kluwer Academic Publishers, Dordrecht, 1991.

[6] Z. G. Li, C. Y. Wen, Y. C. Soh, and W. X. Xie, The stabilization and synchronization of Chua's oscillators via impulsive control, IEEE Trans. Circuits Systems I Fund. Theory Appl. 48 (2001), no. 11, 1351-1355.

[7] G. N. Nair and R. J. Evans, Stabilization with data-rate-limited feedback: tightest attainable bounds, Systems Control Lett. 41 (2000), no. 1, 49-56.

[8] Exponential stabilizability of finite-dimensional linear systems with limited data rates, Automatica 39 (2003), no. 4, 585-593.

[9] I. R. Petersen and A. V. Savkin, Multi-rate stabilization of multivariable discrete-time linear systems via a limited capacity communication channel, Proceedings of the 40th IEEE Conference on Decision and Control, Orlando, 2001, pp. 304-309.

[10]_- State feedback stabilization of uncertain discrete-time linear systems via a limited capacity communication channel, Proceedings of IEEE Conference on Information, Decision and Control, Adelaide, 2002, pp. 211-216.

[11] V. N. Phat, Stabilization of linear continuous time-varying systems with state delays in Hilbert spaces, Electron. J. Differential Equations (2001), no. 67, 1-13.

[12] - New stabilization criteria for linear time-varying systems with state delay and normbounded uncertainties, IEEE Trans. Automat. Control 47 (2002), no. 12, 2095-2098.

[13] Constrained Control Problems of Discrete Processes, Series on Advances in Mathematics for Applied Sciences, vol. 42, World Scientific Publishing, New Jersey, 1996.

[14] V. N. Phat and T. T. Kiet, On the Lyapunov equation in Banach spaces and applications to control problems, Int. J. Math. Math. Sci. 29 (2002), no. 3, 155-166.

[15] D. J. Stilwell and B. E. Bishop, Platoons of underwater vehicles: communication, feedback and decentralized control, IEEE Control Systems Magazine 20 (2000), 45-52.

[16] V. Venkataramanan, K. Peng, B. M. Chen, and T. H. Lee, Discrete-time composite nonlinear feedback control with an application in design of a hard disk drive servo system, IEEE Trans. Contr. Syst. Tech. 11 (2003), 16-23.

[17] W. S. Wong and R. W. Brockett, Systems with finite communication bandwidth constraints. I. State estimation problems, IEEE Trans. Automat. Control 42 (1997), no. 9, 1294-1299. 


\section{Stabilization of discrete-time systems}

[18]_, Systems with finite communication bandwidth constraints. II. Stabilization with limited information feedback, IEEE Trans. Automat. Control 44 (1999), no. 5, 1049-1053.

V. N. Phat: Institute of Mathematics, 18 Hoang Quoc Viet Road, Cau Giay, 10307 Hanoi, Vietnam E-mail address: vnphat@math.ac.vn

J. Jiang: School of Electrical Engineering \& Telecommunications, University of New South Wales, Sydney 2052, Australia

E-mail address: jiangjm@yahoo.com 


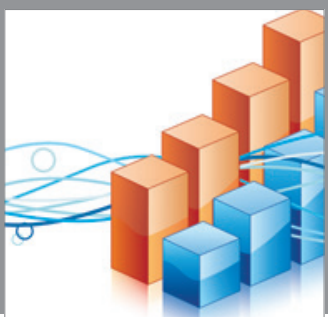

Advances in

Operations Research

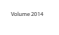

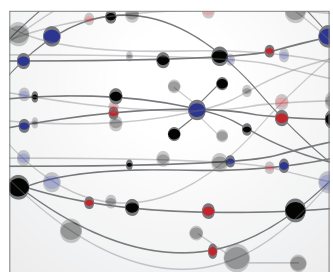

\section{The Scientific} World Journal
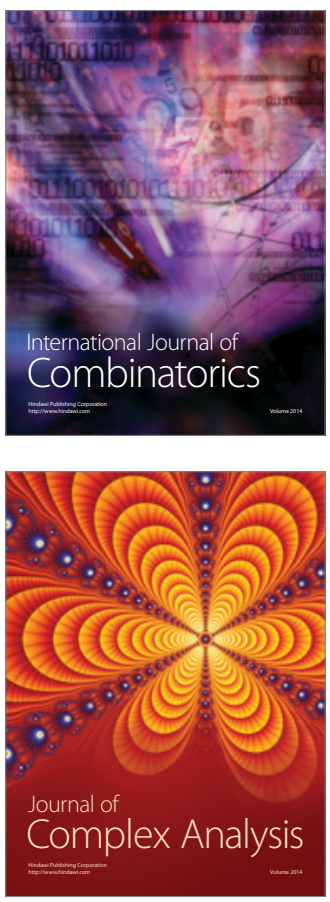

International Journal of

Mathematics and

Mathematical

Sciences
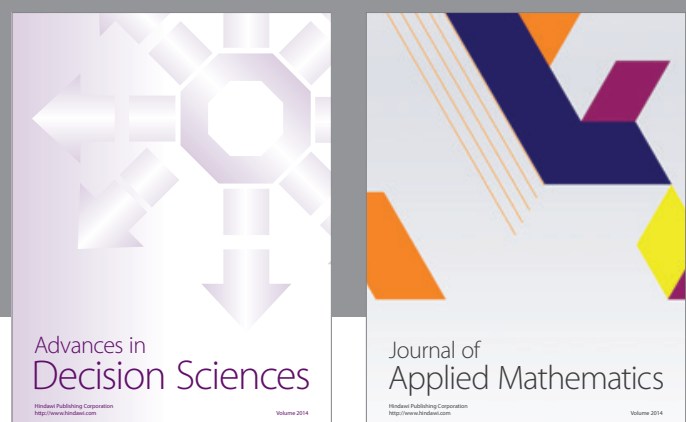

Journal of

Applied Mathematics
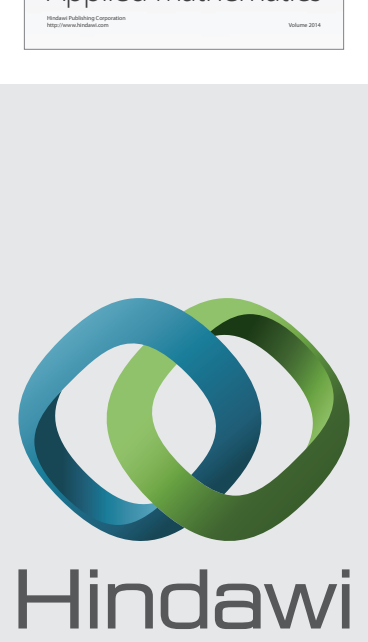

Submit your manuscripts at http://www.hindawi.com
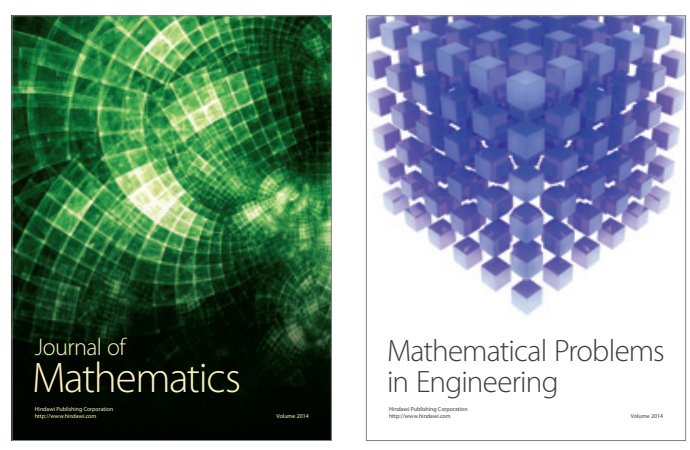

Mathematical Problems in Engineering
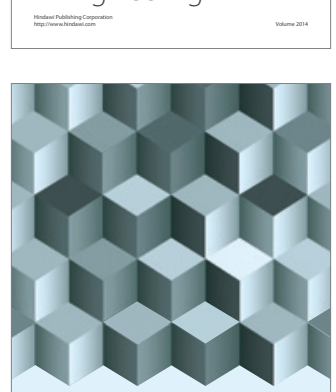

Journal of

Function Spaces
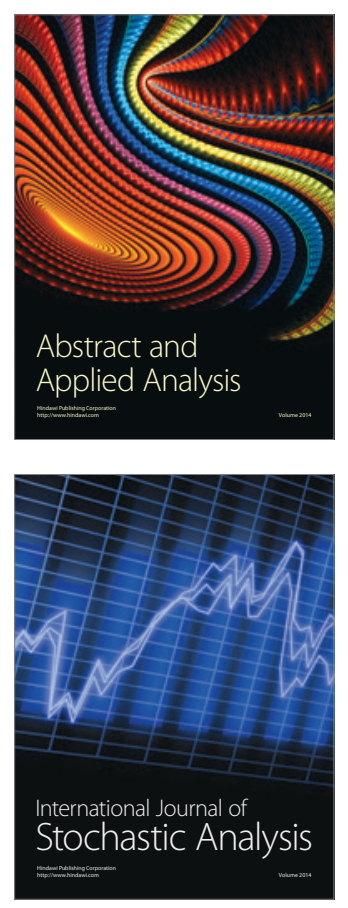

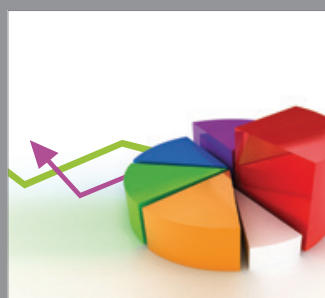

ournal of

Probability and Statistics

Promensencen
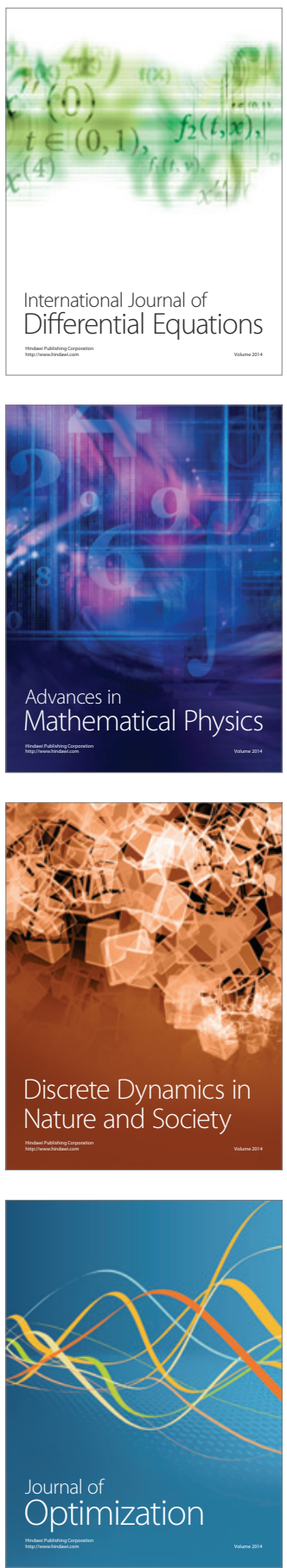